\title{
Influence of the Cozoaltepec River sediment on turtle nesting at La Escobilla beach in Oaxaca, on the Mexican Pacific coast
}

\author{
Mayumy Amparo Cabrera-Ramírez¹, Arturo Carranza-Edwards² y Enrique Quintero de Leonardo ${ }^{3}$ \\ ${ }^{1}$ Departamento de Geología, División de Ingeniería en Ciencias de la Tierra, Facultad de Ingeniería, Universidad Nacional Autónoma de México. Ciudad Universitaria, CDMX, \\ 04510. México \\ ${ }^{2}$ Laboratorio de Sedimentología, Unidad Académica de Procesos Oceánicos y Costeros, Instituto de Ciencias del Mar y Limnología, Universidad Nacional Autónoma de \\ México. Ciudad Universitaria, CDMX, 04510. México \\ ${ }^{3}$ Posgrado en Ciencias del Mar y Limnología, Universidad Nacional Autónoma de México. Ciudad Universitaria, CDMX, 04510. México \\ e-mail: mayumycr@unam.mx \\ Recibido: 18 de enero de 2017. \\ Aceptado: 26 de octubre de 2017.
}

Cabrera-Ramírez M. A., A. Carranza-Edwards y E. Quintero de Leonardo. 2018. Influencia del aporte sedimentario del río Cozoaltepec en la anidación de tortugas en la playa La Escobilla en Oaxaca, en el Pacífico mexicano. Hidrobiológica 28 (1): 71-81 D0l: 10.24275/uam/izt/dcbs/hidro/2018v28n1/Cabrera

\section{RESUMEN}

Antecedentes. La Escobilla es una playa arenosa que constituye el principal sitio de anidación de la tortuga golfina (Lepidochelys olivacea) en la costa mexicana. Los estudios relacionados con el hábitat de estos organismos permiten establecer estrategias de conservación dentro de las cuales es necesario conocer los parámetros físicos que afectan las arribazones. Objetivos. Este estudio determinó las características texturales de los sedimentos litorales y la morfología de la playa con el fin de evaluar sus posibles relaciones con las anidaciones de esta especie. Métodos. Se colectaron muestras de sedimento en las zonas de infraplaya, mesoplaya y supraplaya a lo largo de $15 \mathrm{~km}$ de playa; se realizaron levantamientos topográficos de perfiles de playa, y se obtuvieron los parámetros texturales de Mz $\phi$ (tamaño gráfico promedio) y $\sigma \phi$ (clasificación gráfica inclusiva). Resultados. Las zonas de mesoplaya y de supraplaya con pendiente suave del orden de $2^{\circ}$ a $4^{\circ}$, tamaño de grano de arenas medias $(1 \phi$ a $2 \phi)$ y la apertura de la barra arenosa por la desembocadura del río Cozoaltepec en época de lluvias parecen favorecer las anidaciones. Conclusiones. Las zonas con mayores sitios de anidación se encuentran a una distancia de $2.3 \mathrm{~km}$ hacia el este del río Cozoaltepec, donde aparentemente la morfología de la playa es más adecuada, ya que su pendiente no es demasiado alta y la playa es suficientemente amplia para las anidaciones. Además, en esa localidad se presentó el mayor número de arribazones de las tortugas.

Palabras clave: análisis textural, arribazón, perfil de playa, playa La Escobilla, tortuga golfina

\begin{abstract}
Background. La Escobilla is a sandy beach and the main nesting site of the Olive Ridley turtle, Lepidochelys olivacea, (golfina in Spanish), from the southern Mexican coast. Studies related to the habitat of these organisms suggest conservation strategies that lead us to examine the physical parameters that affect the arribazones [i.e., coastal abundance of fish, off-shore shoals, and, by extension, abundance of turtles]. Goals. The aim of this study was to investigate the textural characteristics of coastal sediments and morphology of the beach in order to determine their possible relation with the nesting of this species. Methods. Sediment samples were collected along $15 \mathrm{~km}$ of infra-, meso-, an supra-beach, topographic surveys of beach profiles were made, and some sedimentological parameters were obtained, i.e., Mzф (size grain) and $\sigma \phi$ (sediment classification). Results. Meso-beach and supra-beach areas with a gentle slope (from $2^{\circ}$ to $4^{\circ}$ ), grain size medium sands ( $1 \phi$ to $2 \phi)$, and the opening of the sandbar at the mouth of the Cozoaltepec River during rainy season appear to favor nesting. Conclusions. Areas with major nesting sites are located at a distance of $2.3 \mathrm{~km}$ to the east of the Cozoaltepec River, where apparently the morphology of the beach is more adequate because its slope is not so high and the beach is wide enough for nesting. We also know that the largest observed turtle arribazones occur in this area.
\end{abstract}

Keywords: beach profile, La Escobilla beach, Olive Ridley, textural analysis, turtle arribazones 


\section{INTRODUCCIÓN}

La Escobilla es una playa ubicada en el Pacífico mexicano, en el estado de Oaxaca. Su importancia ecológica se debe a que es el centro de anidación más importante de México para la tortuga marina Lepidochelys olivacea (Eschschotlz, 1929), conocida con los nombres de tortuga golfina, oliva o lora. Esta playa presenta las mayores arribazones en playas oaxaqueñas; entendiendo como arribazón el fenómeno de anidaciones en grandes agregados (Ávila, 2006).

Lepidochelys olivacea es una de las siete especies de tortugas marinas (Fig. 1) que existen actualmente en el mundo y se les encuentra en los mares tropicales y subtropicales de América (Eckert et al., 1999). Esta especie está catalogada como vulnerable tanto por la Unión Internacional para la Conservación de la Naturaleza (IUCN, 2015) como por la legislación mexicana en la NOM-059-Semarnat-2001 (Seminoff \& Shanker, 2008). En las últimas décadas se han realizado esfuerzos para promover la conservación y protección tanto de los organismos como de sus hábitats, lo cual ha resultado en el incremento en el número de anidaciones para varias poblaciones (Hays, 2004; Seminoff \& Shanker, 2008). En México se tiene el Programa Nacional de Protección, Conservación, Investigación y Manejo de Tortugas Marinas, el cual coordina 31 centros para la conservación de las tortugas marinas (CPCTM) distribuidos en varias entidades costeras del país. Los CPCTM son centros de protección y conservación donde se llevan a cabo planes de manejo y de recuperación de las poblaciones de las especies de tortugas marinas de México, es decir, se protegen tanto los nidos como las tortugas, lo que propicia un mayor éxito en su eclosión (CONANP, 2008).

Los sitios de anidación de las tortugas marinas son las playas arenosas. Este ambiente se puede definir como el lugar donde se acumula el material suelto que está en los alrededores del límite de acción del oleaje (King, 1972). Es un ambiente muy dinámico afectado por corrientes litorales, mareas, vientos y por aportaciones de sedimen- tos fluviales; por lo tanto, presenta gran variabilidad en su expresión morfológica, así como en sus parámetros sedimentológicos (Carranza, 1984, 2001). Dentro de los parámetros que pudieran ser utilizados por las tortugas para sus sitios de anidación, podrían estar los siguientes: humedad del sedimento, tamaño de grano, perfil de playa, temperatura de la arena y las anomalías magnéticas, según Mortimer $(1990,1995)$, Wood y Bjorndal (2000) y Fuentes-Farías et al. (2010).

Las características de las playas de anidación varían en función de las especies de tortugas marinas (Eckert et al., 1989; Mortimer, 2000; Pritchard \& Mortimer, 2000). Para el caso de la playa La Escobilla, no hay un estudio a fondo que permita conocer la relación entre estos organismos y el entorno geológico en el cual realizan sus anidaciones. Este trabajo tiene como objetivo investigar las características texturales de los sedimentos litorales y la morfología de la playa para determinar sus posibles relaciones con el arribazón de la tortuga golfina.

La playa La Escobilla se encuentra en el Pacífico sur mexicano, sobre las costas del estado de Oaxaca, entre las coordenadas $15^{\circ} 47^{\prime}$ $18^{\prime \prime} \mathrm{N}$, longitud $96^{\circ} 56^{\prime} 54^{\prime \prime} 0$ y latitud $15^{\circ} 44^{\prime} 39^{\prime \prime} \mathrm{N}$ y longitud $96^{\circ} 51^{\prime}$ $57^{\prime \prime} 0$ (Fig. 2). En esta playa, ubicada en el municipio de Santa María Tonameca, distrito de Pochutla, se encuentra un centro tortuguero. Esta zona forma parte de la zona marina prioritaria número 34 , denominada Chacahua-Escobilla y tiene una extensión de $615 \mathrm{~km}^{2}$ (Arriaga et al., 1998). La playa tiene una longitud de $12 \mathrm{~km}$, entre los que se encuentran distintos humedales. Al poniente se localiza la desembocadura del río Cozoaltepec y la barra de Potrero, y al oriente se encuentra la barra de Tilaza. Entre estas desembocaduras se encuentran pequeños humedales; de poniente a oriente: barra de Salinas, barra Escobilla y barra de Macahuite. Estos pequeños humedales drenan hacia el mar en temporada de lluvias y mareas altas (Albavera, 2006). El campamento tortuguero está localizado en las coordenadas $15^{\circ} 43^{\prime} 66^{\prime \prime} \mathrm{N}$ y $96^{\circ} 45^{\prime}$ $67 " 0$, con acceso situado a la altura del kilómetro 180.5 de la carretera federal 200, entre las poblaciones de Puerto Escondido y Pochutla.

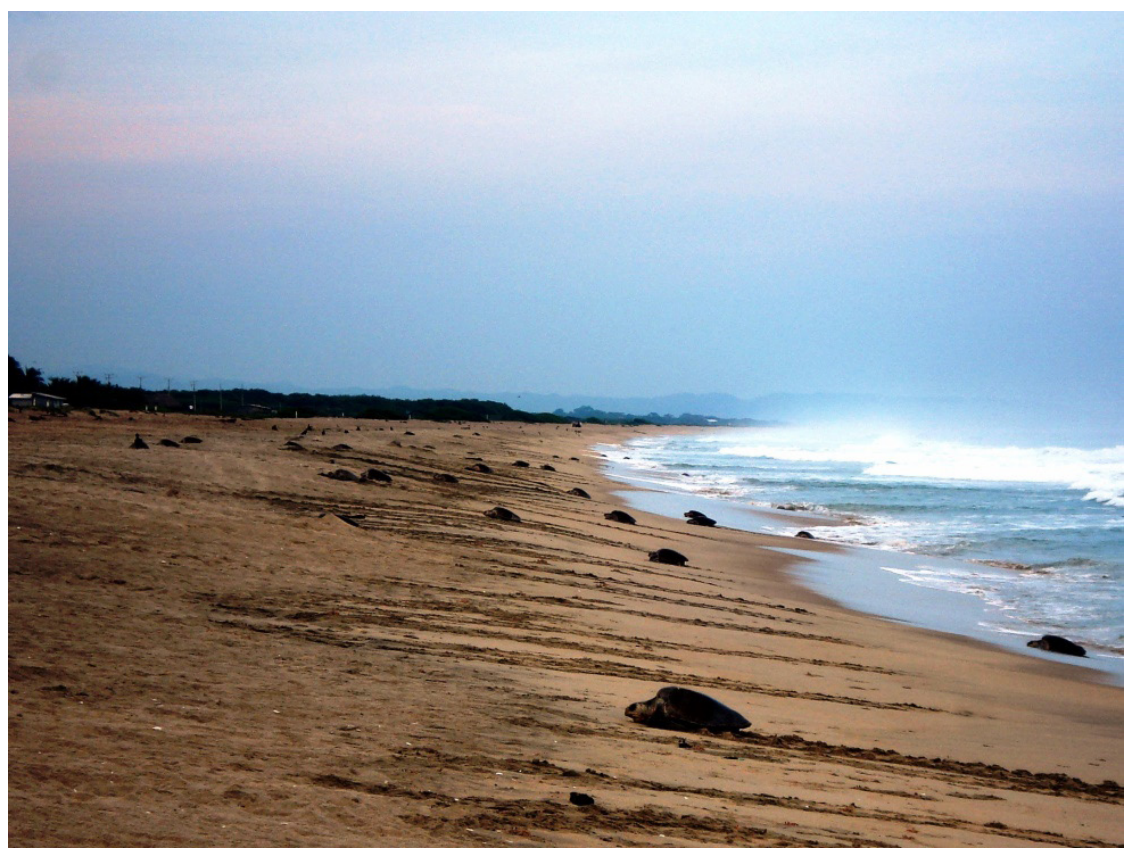

Figura 1. Arribazón de tortuga golfina (Lepidochelys olivacea (Eschschotlz, 1929)) en la playa La Escobilla (Foto: Arturo Carranza-Edwards). 


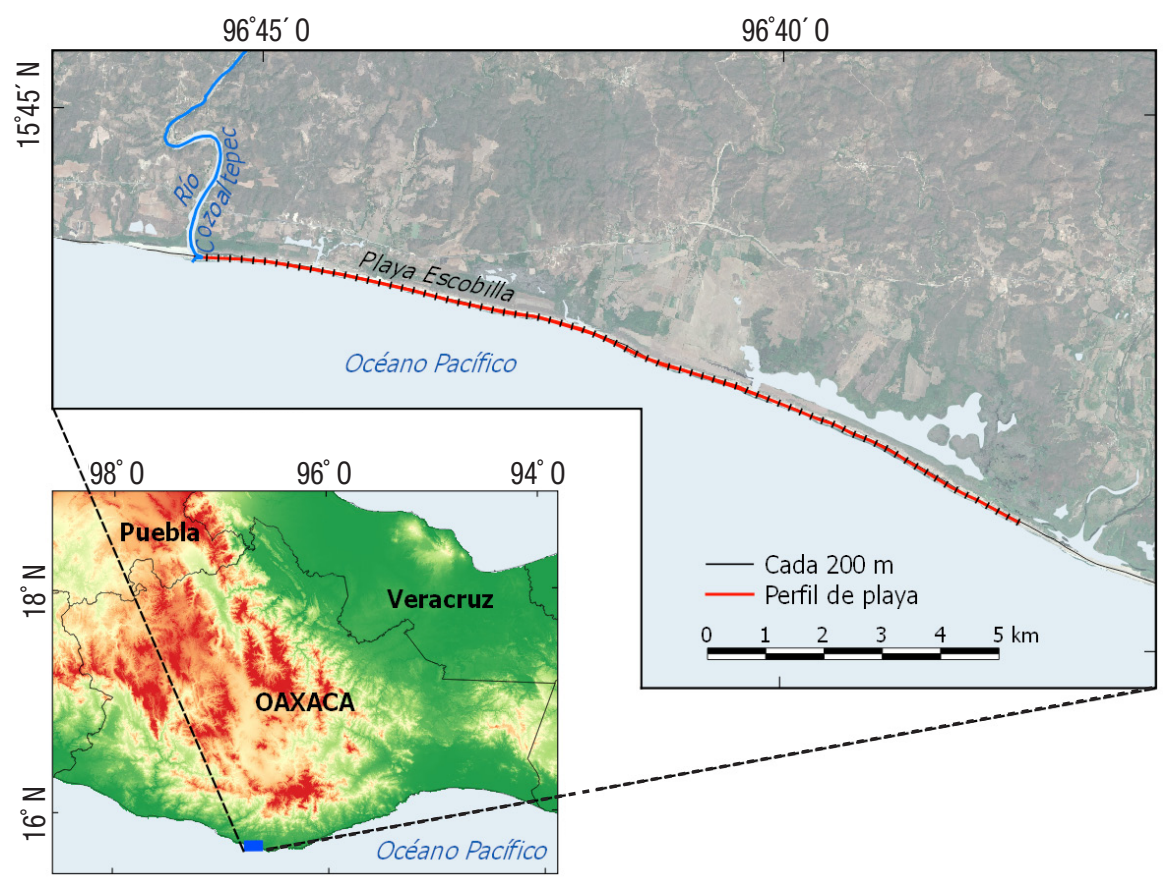

Figura 2. Área de estudio. Playa ubicada al Este de la desembocadura del río Cozoaltepec, Oaxaca, México.

El ancho promedio de la playa es de $44.28 \mathrm{~m}$, aunque disminuye de poniente a oriente, con un máximo a dos kilómetros de distancia de la barra del río Cozoaltepec con $59 \mathrm{~m}$ y un mínimo de $24.6 \mathrm{~m}$ a 200 $\mathrm{m}$ de la barra de Macahuite; no obstante, el perfil de playa se amplía cerca de las barras. El intervalo de marea es menor que $2 \mathrm{~m}$ hacia el oeste de Salina Cruz y de 2 a $4 \mathrm{~m}$ hacia el este del mismo puerto. En ambos casos, la marea es de tipo mixto (Davies, 1973). De acuerdo con las cartas mensuales de corrientes superficiales (Wyrtki, 1965), las próximas a las costas oaxaqueñas presentan en promedio velocidades menores que $0.15 \mathrm{~m} / \mathrm{s}$, con un máximo de $0.21 \mathrm{~m} / \mathrm{s}$ medido en el mes de junio. Del mes de mayo al mes de septiembre la dirección de estas corrientes es principalmente de Este a Oeste, en tanto que el resto del año se presenta al oeste de Puerto Ángel una corriente Este-Oeste y al este del mismo puerto la corriente tiene una dirección Oeste-Este (Carranza et al., 1987).

Tectónicamente, el litoral de 0axaca forma parte de la costa de colisión continental, afectada por el contacto de la placa de América con la placa de Cocos (Carranza et al., 1975). Fisiográficamente, el área de estudio pertenece a la provincia Sierra Madre del Sur, dentro de Ios terrenos tectono-estratigráficos Oaxaca y Xolapa (Lugo, 1990). Las rocas que afloran en las cuencas hidrológicas que drenan hacia el mar son de litología y edad muy diversa (Fig. 3). En las estaciones de playa estudiadas se reciben principalmente los aportes de sedimentos procedentes de las rocas metamórficas (gneises y anortositas) que constituyen el complejo 0axaqueño (pTmCM); gneises cuarzo feldespáticos, anfibolitas, pegmatitas migmatitas y algunos horizontes de mármol que forman al complejo Xolapa, y sedimentos procedentes de las rocas intrusivas que afectaron al complejo Xolapa y que afloran en la región, de edad terciaria y de composiciones que varían de granito a granodiorita (Servicio Geológico Mexicano, 2002). Las rocas cuaternarias que afloran en la zona están representadas por el conglomerado Puerto Escon- dido (QptCgp), formado por una alternancia de conglomerado polimíctico y arenas poco consolidadas, así como depósitos palustres (Qhopa), de litoral (Qholi) y aluviones (Qhoal).

\section{MATERIALES Y MÉTODOS}

En época de lluvias el río Cozoaltepec rompe la barra arenosa, lo que permite el libre flujo de agua dulce hacia el mar y la distribución de arenas desde el oeste del río hacia el este, pues al oeste se encuentra un litoral rocoso que en parte funciona como una barrera que favorece que la anchura de la playa sea más amplia hacía el Este.

En el oeste de la desembocadura del río Cozoaltepec no se muestreó la playa, ya que en esa porción no se presentaron sitios de arribazón de tortugas en septiembre de 2007. Se muestrearon $15 \mathrm{~km}$ de playa al este de la desembocadura del río, en transectos divididos cada $200 \mathrm{~m}$ en los primeros $5 \mathrm{~km}$, cada $1000 \mathrm{~m}$ los siguientes $5 \mathrm{~km}$, y al final a $5000 \mathrm{~m}$; dado que, a medida que se aleja uno de la desembocadura del río, disminuye el ancho de la playa y desaparecen los sitios de anidación. El último muestreo de arena se realizó a $15 \mathrm{~km}$ al este de la desembocadura del río, para comparar sitios sin anidaciones con los sitios donde sí las hay (Fig. 3). En cada lugar se tomaron tres muestras de sedimento superficial; la primera de la infraplaya, la segunda de mesoplaya y la tercera en supraplaya, subzonas así denominadas por Carranza-Edwards y Caso-Chávez (1994). En cada transecto de la playa arenosa se realizó el levantamiento topográfico de los perfiles con el método de estadal y telémetro (Figs. 4a-l; 5a-n).

Con la finalidad de obtener los parámetros tamaño gráfico promedio y desviación estándar, propuestos por Folk (1974), se realizó una separación de tamaños de partículas mediante tamizado de arenas y posteriormente las partículas finas (lodos) con contador láser de partículas. 


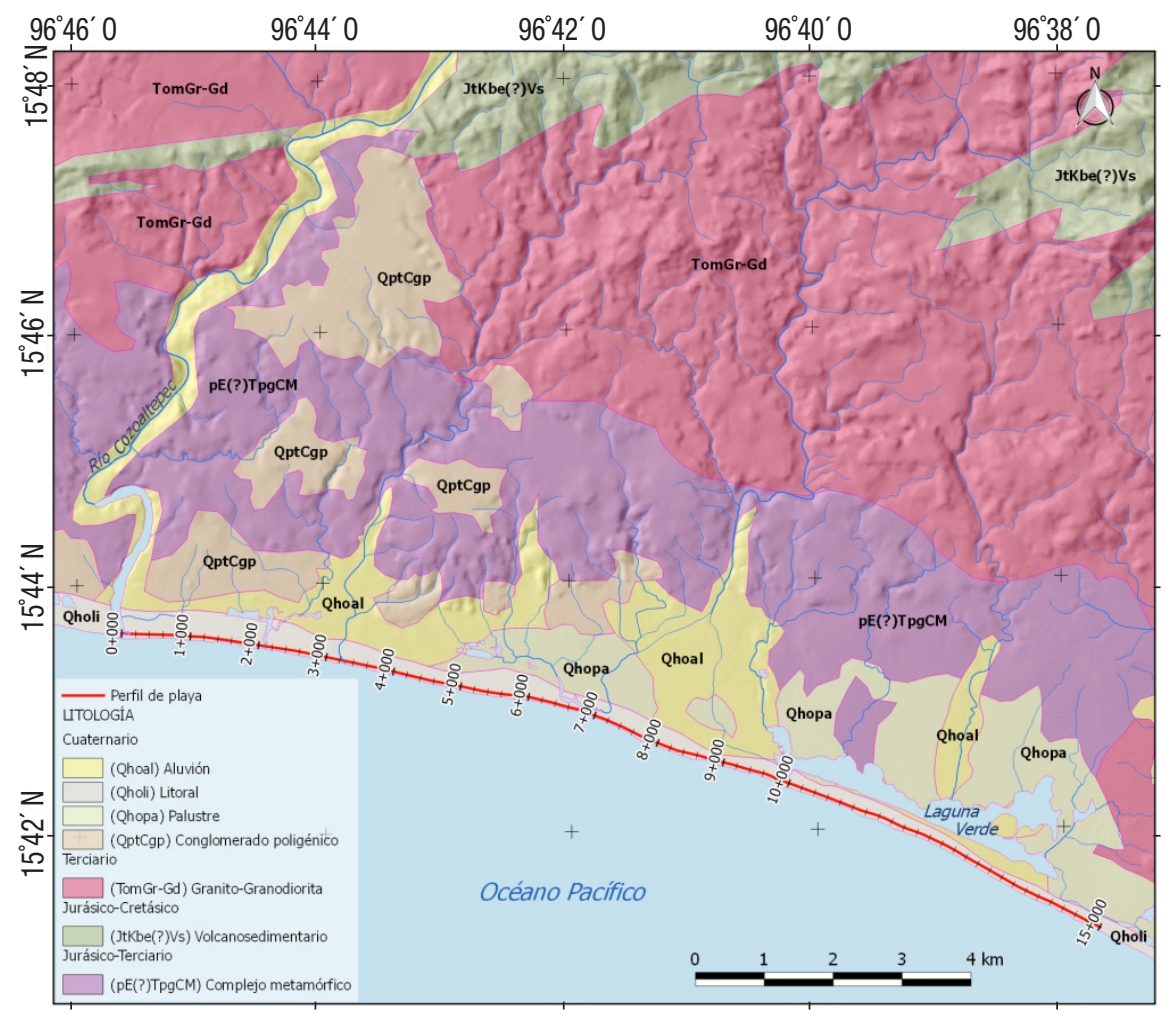

Figura 3. Mapa geológico de la región de la desembocadura del río Cozoaltepec, Oaxaca, México (modificado del Servicio Geológico Mexicano, 2002), en el cual se aprecia la línea roja que señala los sitios donde se levantaron los perfiles. Los números se refieren a la distancia en kilómetros desde la desembocadura del río Cozoaltepec.

\section{RESULTADOS}

Morfología de la playa. La morfología de la playa (ancho, pendiente, cambios en la línea de costa) ha sido considerada como un importante factor en la selección de sitios de anidación de la tortuga golfina (Bolongaro et al., 2010; Barik et al., 2014). Los cambios en la morfología costera provocados por la erosión, tanto del oleaje como de tormentas y huracanes, deben de ser monitoreados para tener un manejo sustentable de los litorales (Pethick \& Crooks, 2000). El análisis de los perfiles de playa en el área de estudio indica que a lo largo de la playa La Escobilla el ancho de la playa disminuye considerablemente conforme se incrementa la distancia a partir de la desembocadura del río Cozoaltepec.

Las estaciones más cercanas a la desembocadura del río tienen un ancho de playa que varía desde 60 a $80 \mathrm{~m}$ y hasta un mínimo de $23 \mathrm{~m}$ a los $5 \mathrm{~km}$ hacia el este de la desembocadura del río. Estas variaciones presentan una relación inversa en los primeros $5 \mathrm{~km}$ al este de la desembocadura con respecto al ángulo de playa, por lo que se observa que la playa es más ancha y el ángulo de la playa es menor en esta zona, y se encuentra que la anidación es favorecida cuando la playas es ancha $(70 \mathrm{~m})$ pero la pendiente es pequeña (entre 2 y $4^{\circ}$ ), lo que facilita el ascenso de las tortugas durante el proceso de anidación (Fig. 6).

La disminución en el ancho de playa está relacionada con el aporte de material sedimentario procedente del río Cozoaltepec. La carga de sedimentos arenosos procedentes del río es suficiente para alimentar y mantener un ancho de playa mayor que $50 \mathrm{~m}$ hasta los $5 \mathrm{~km}$ a partir de la desembocadura, que son transportados con dirección oeste-este. Después de los $5 \mathrm{~km}$ se observa una disminución en el ancho de playa hasta llegar a oscilar entre los 30 y 40 metros. Se observó que, aunado a la disminución del ancho de playa, la presencia de cuspilitos es más frecuente. Al disminuir el ancho de playa, se aprecia un decremento en el número de nidos a partir de los $5 \mathrm{~km}$ al este de la desembocadura del río Cozoaltepec, indicio de que la zona de playa a partir de los $5 \mathrm{~km}$ empieza a ser menos adecuada para las anidaciones, donde se observa que las pendientes oscilan entre 4 y $8^{\circ}$ y el ancho de playa entre los 30 y 45 m (Fig. 6).

Análisis textural. Para la conservación de especies es necesario establecer estrategias, para las cuales es preciso conocer las características de la arena de las playas de anidación; ya que se ha observado que las diferencias entre el tamaño de grano, las composiciones mineralógica (Mortimer, 1990; Schwartz, 1982) y química (Johannes \& Rimmer, 1984; Fuentes-Farías et al., 2010) de la arena influyen en el éxito de la eclosión.

Las características del medio determinarán la variedad de ambientes de incubación, que incidirán directamente en proporcionar diferentes rasgos, como el sexo de las crías de tortugas, por lo que las estrategias de conservación que incluyan el movimiento de los nidos de mesoplaya a supraplaya reducirían esta variación en los ambientes, con lo que disminuiría la posibilidad de contar con diferentes características que promuevan el éxito en la eclosión (Foley et al., 2006; Mazaris et al., 2009). 
El análisis textural de las tres zonas que conforman la playa La Escobilla (infraplaya, mesoplaya y supraplaya) muestra que el tamaño de grano en la de infraplaya a lo largo de La Escobilla es heterogéneo, dado que en esa zona se observan arenas medias ( $1 \phi$ a $2 \phi)$ cercanas a la desembocadura del río Cozoaltepec, que gradúan a arenas gruesas $(<1 \phi)$ hacia el primer kilómetro en dirección al este para posteriormente cambiar a arenas medias después del kilómetro 2, graduar nuevamente a arenas gruesas entre el kilómetro 3 y 6 y a partir de aquí arenas medias hasta el kilómetro 9 (Figs. 7a-c, 8a-c)). Foley et al. (2006) encontraron que el tamaño de grano promedio de las arenas de playa en las que se tiene éxito en la eclosión corresponde con la arena media (1申); para el caso de la playa La Escobilla se observan resultados similares, donde las anidaciones se dan por igual en la mesoplaya y supraplaya. En estas zonas se tiene que a lo largo de toda la playa el tamaño de grano promedio es de arenas medias $(1 \phi)$, donde se observa una tendencia de los sedimentos a ser homogéneos conforme se sube en el perfil de la playa, desde la infraplaya hasta la supraplaya. Esto parece indicar que la propia bioturbación producida por las tortugas en meso y supraplaya influye en la homogeneización del tamaño de grano $(M z \phi)$. En dirección paralela a la línea de costa no se observa alguna tendencia, ni hacia los tamaños finos ni hacia los tamaños gruesos.
Los valores de clasificación o desviación gráfica inclusiva indican que para la zona de infraplaya varían desde $0.9 \sigma \phi$ a $0.5 \sigma \phi$; es decir, que se pueden encontrar arenas que varían desde moderadamente clasificadas a moderadamente bien clasificadas, mientras que en las zonas de mesoplaya y supraplaya las muestras se encuentran moderadamente bien clasificadas.

Las variaciones en el tamaño de grano con respecto a la subzona de playa están directamente relacionadas con la energía del transporte que predomina en cada uno. Dentro de la infraplaya, el agente de transporte es hidráulico, pues es la acción del oleaje y de las corrientes tanto litorales como de retorno lo que pone en movimiento al sedimento; por lo tanto, el tamaño de grano tiende a ser heterogéneo. Por el contrario, el viento es el agente que transporta el material, tanto en mesoplaya como en supraplaya, al transportar granos de tamaños más finos que en infraplaya (Figs. 8a-c)).

El viento es un agente de transporte selectivo, pues en condiciones normales no tiene la capacidad de transportar material muy pesado, y ello influye en el comportamiento de la textura del sedimento en ambas subzonas. El comportamiento de los parámetros estadísticos por subzonas a lo largo de la playa coincide con el reportado por Carran-
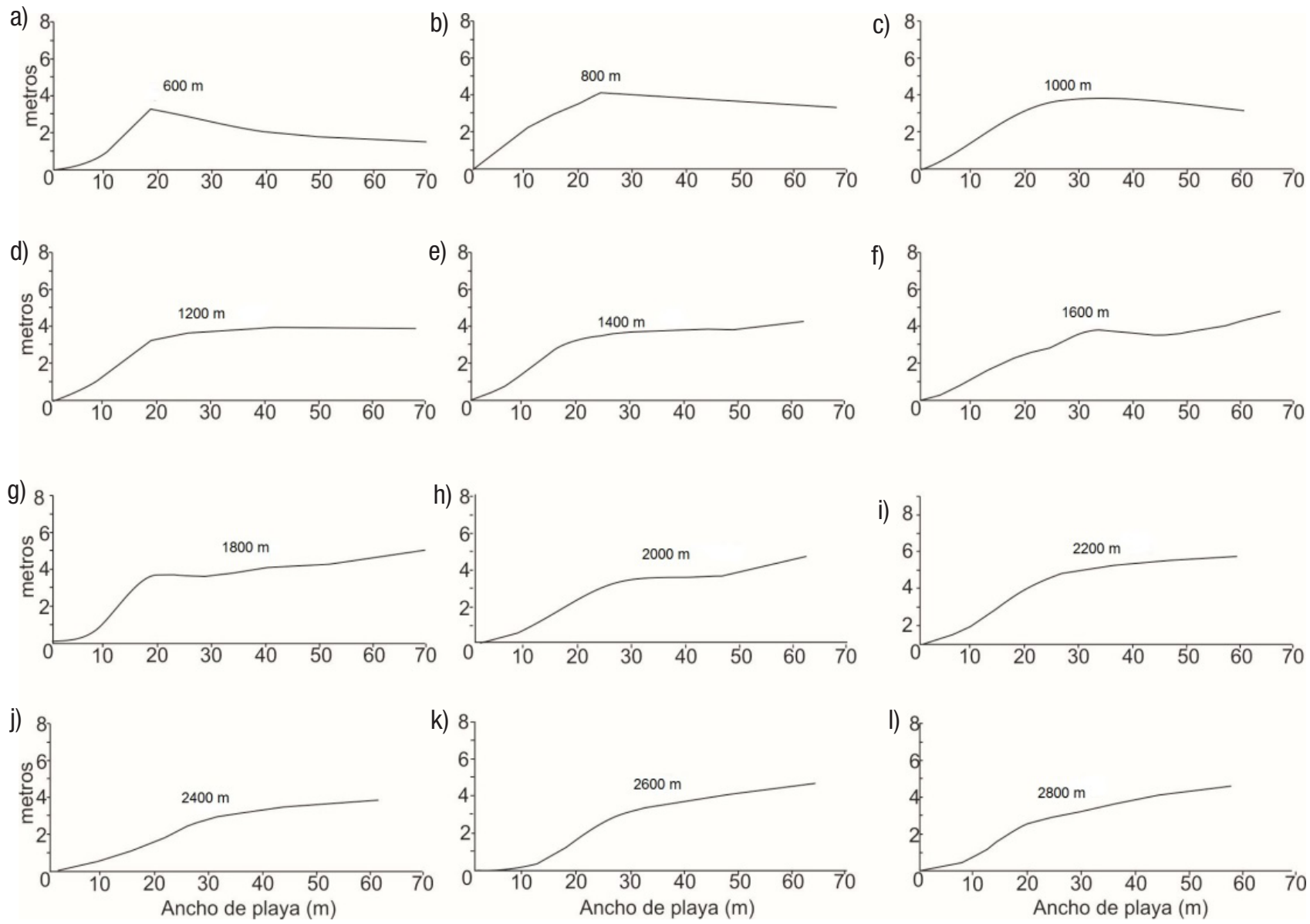

Figuras 4a-I. Perfiles de playa cada 200 m, a partir de 600 m de la desembocadura del río Cozoaltepec, Oaxaca, México, hasta 2800 m hacia el Este. 
za (2001) y Komar (1976); sin embargo, en este caso los parámetros estadísticos se homogenizan en mesoplaya y supraplaya, debido a la bioturbación generada por la gran cantidad de tortugas que mezclan la arena de estas dos subzonas.

\section{DISCUSIÓN}

El tamaño de grano promedio de las arenas de playa en las que se tiene éxito en la eclosión es de arena media (1ф), aunque para especies como Caretta caretta (Linnaeus, 1758) el tamaño promedio puede ser de arenas finas $(2 \phi$ a $3 \phi)$ (Schwartz, 1982). Para el caso de La Escobilla, y particularmente para la especie Lepidochelys olivacea, en las zonas de mesoplaya y supraplaya se presentan arenas medias $(1 \phi$ a $2 \phi)$ de moderadamente clasificadas a moderadamente bien clasificadas, resultados similares a los encontrados en la India para sitios de anidación de tortuga golfina (Barik et al., 2014).

La selección de sitios de anidación también puede estar en función de la salinidad; en ese sentido, las arenas con menor salinidad son los a)

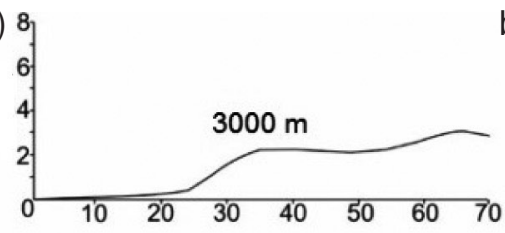

b)

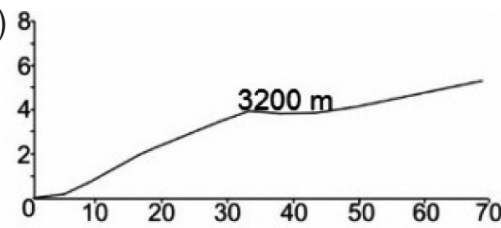

c)

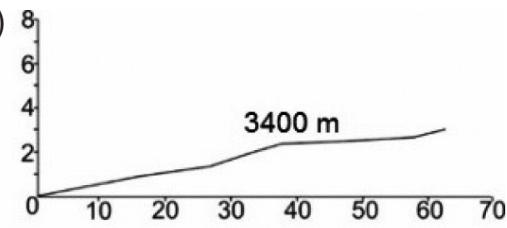

d)

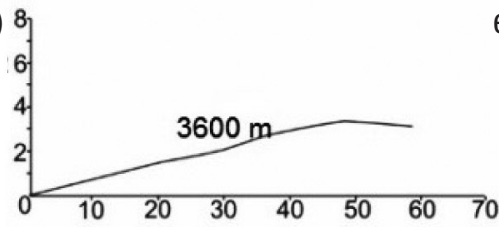

e)

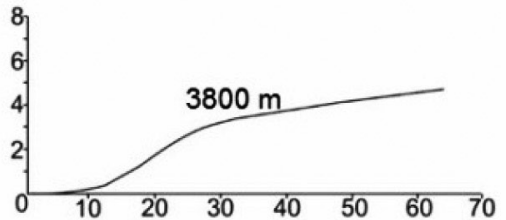

f)

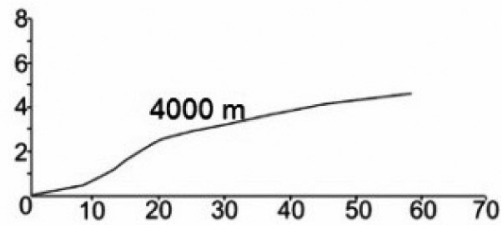

g) 8

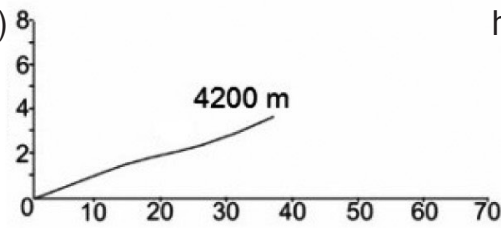

h)

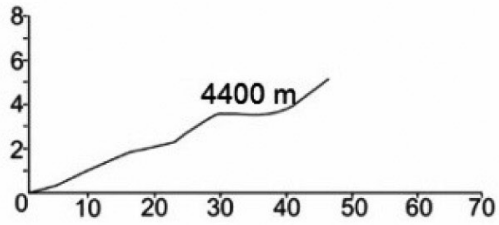

i)

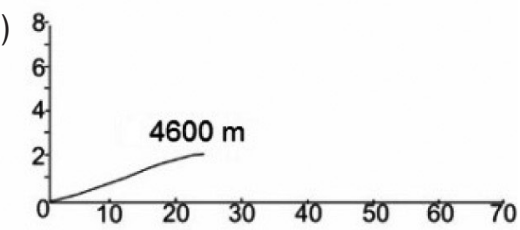

j)

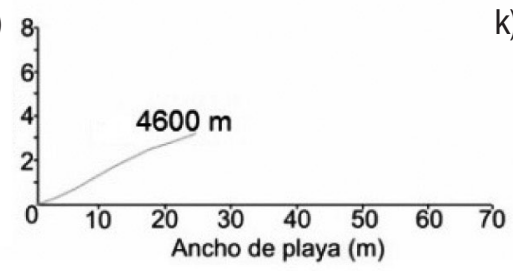

k)

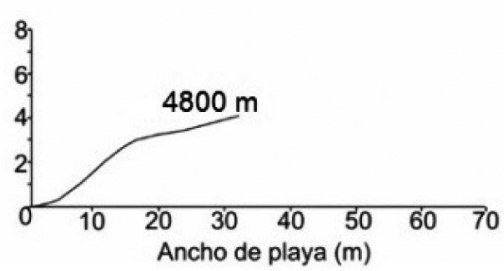

l)

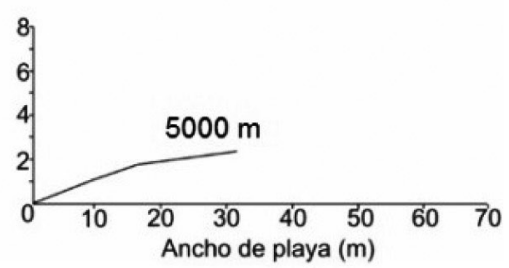

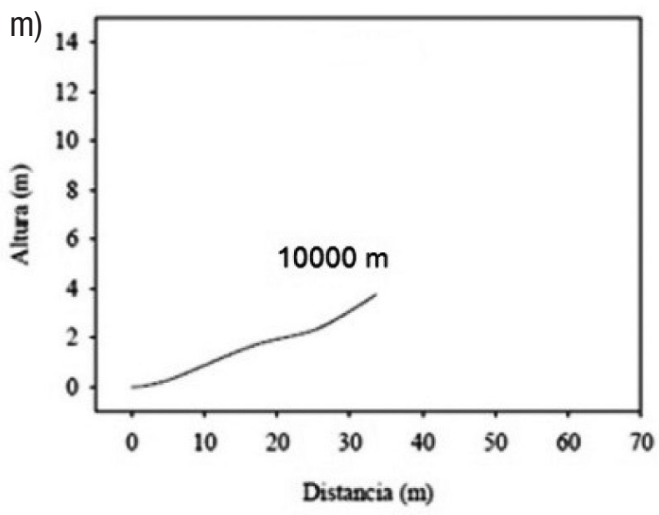

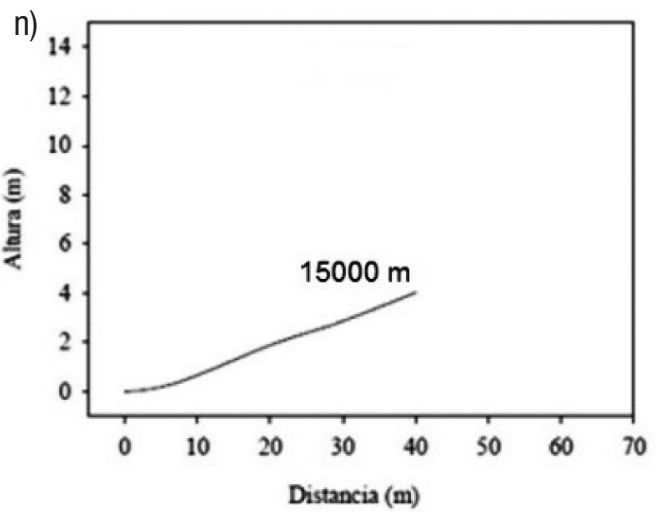

Figuras 5a-n. Perfiles de playa cada 200 m, a partir de 3000 m de la desembocadura del río Cozoaltepec, Oaxaca, México, hacia el Este hasta 5000 m. Un perfil de playa levantado a los $10000 \mathrm{~m}$ y otro a $15000 \mathrm{~m}$. 


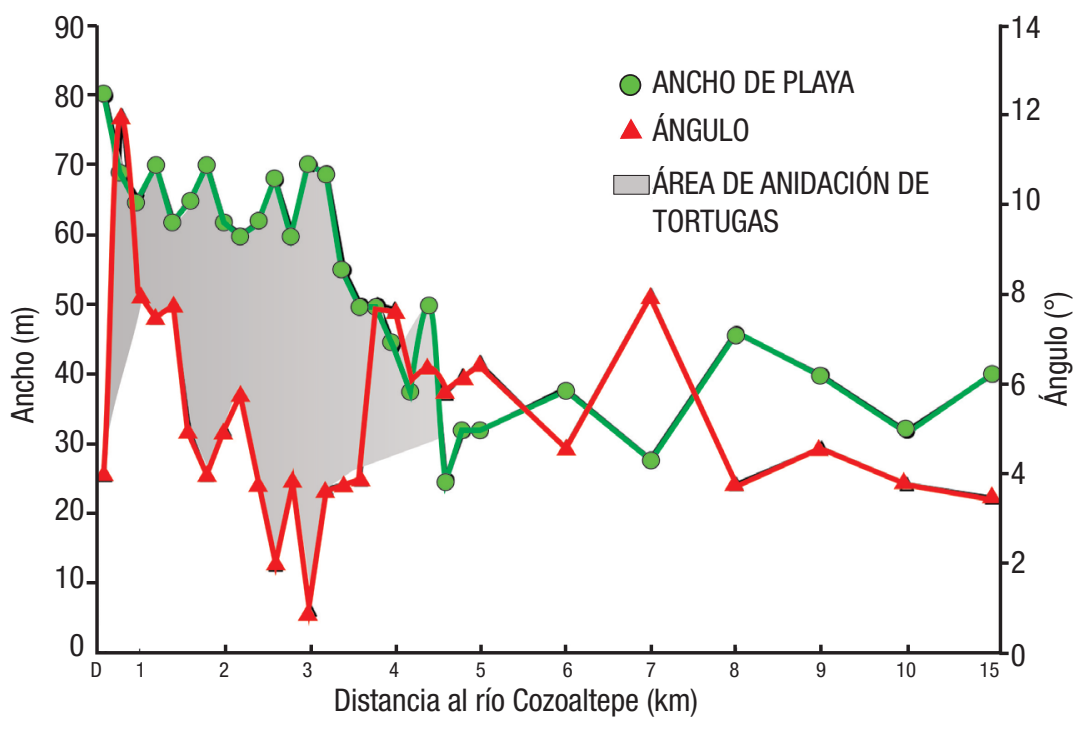

Figura 6. Cambios en el ancho de la playa y en el ángulo de frente de playa La Escobilla, desde la desembocadura del río Cozoaltepec, Oaxaca, México, hasta $15 \mathrm{~km}$ hacia el Este. $\mathrm{D}=$ Desembocadura.

lugares preferidos por las tortugas (Wood \& Bjorndal, 2000), lo cual sucede en la época de lluvias, pues la humectación asociada facilita la excavación en la playa. Es probable que la ruptura de la barra del río por aumento de la precipitación pluvial marque el aviso para el desove, pues en la memoria de las tortugas podrían quedar registradas variaciones de temperatura, disminución de salinidad en la columna de agua y aumento de la descarga de sedimentos hacia el mar (Wood \& Bjorndal, 2000; Poloczanska et al., 2009). Por esa razón se debe evitar algún represamiento del río Cozoaltepec, ya que eso haría que las partículas arenosas queden retenidas principalmente en el embalse. Bajo estas condiciones, en la desembocadura del río prevalecerían sedimentos lodosos, los cuales no permanecen en el litoral, y como consecuencia, se daría el adelgazamiento 0 desaparición de la playa.

Al estudiar el tamaño de grano de las arenas de los sitios de anidación de tortugas marinas, se observa una relación entre el incremento del tamaño de grano y el decremento en el éxito en la eclosión; por un lado, asociado con condiciones muy secas, donde los granos gruesos no retienen el agua necesaria para la anidación (Ackerman, 1997), y, por el otro, relacionado con un decremento en la salinidad asociada con las inundaciones de estos sitios, la cual se produce en épocas de lluvias por el aporte de agua dulce. Sin embargo, la efectividad en el lavado disminuye en la medida que el tamaño de grano decrece (Foley et al., 2006; Yalcin-Özdilek et al. 2007; Foley et al., 2014). Las arenas gruesas, según estos autores, están relacionadas con la abundancia de biógenos de composición carbonatada, pero para el caso de La Escobilla, en donde las condiciones de alta energía generadas por el oleaje observado durante el muestreo hace que la abundancia de estos componentes en las arenas sea mínima o carezca de biógenos; por lo tanto, las arenas gruesas se pueden deber a la cercanía de las rocas fuentes, lo cual es frecuente en el Pacífico, particularmente en las puntas rocosas y más si se trata de granitos y gneises (Carranza-Edwards et al., 2009).
La presencia de los ríos es un factor importante, ya que algunas investigaciones han propuesto que la cercanía con las desembocaduras proporciona tanto una fuente de nutrientes continua (Foley et al., 2006; Hawkes et al., 2009) como una estabilidad física relacionada con la estratificación entre el agua más salada en el fondo y menos salada hacia la superficie, y que permite flotar a la tortuga golfina durante la etapa de copulación anterior a la anidación (Miller, 1997; Barik et al., 2014). Por esta razón, la mayoría de las anidaciones se presentan cercanas al río Cozoaltepec, el cual aporta sedimentos a la playa. Si no hay sedimento, no hay playa donde puedan arribar las tortugas para el desove. La ausencia de sedimentos litorales en las playas se puede originar por causas antrópicas tales como la retención de sedimentos por represamiento ya mencionada), por el desvío de corrientes fluviales o bien por extracción de arenas de playa como materiales de construcción en los sitios de anidación o cercanos a ellos.

El ángulo de la playa es muy importante, ya que las tortugas prefieren playas con pendiente suave (rango entre 2 a $4^{\circ}$ ), debido a que las playas más inclinadas inciden en la probabilidad de un mayor esfuerzo para la anidación de las tortugas. Las playas con pendiente fuerte $\left(8^{\circ}\right)$ son propensas a la erosión y las playas planas (pendiente menor que $1^{\circ}$ ) son propensas a la inundación y a las depredaciones, por ende, las pendientes suaves $\left(2 \mathrm{a} 4^{\circ}\right)$ favorecen las anidaciones, como se encontró en este estudio y también en Barik et al. (2014). En La Escobilla se tienen pendientes que favorecen las anidaciones, cercanas al río Cozoaltepec, en particular, en áreas intermedias de la playa donde la pendiente no es muy pronunciada $\left(2 \mathrm{a} 4^{\circ}\right)$ como sucede cerca de su desembocadura.

Las principales anidaciones se dan cerca de la desembocadura del río Cozoaltepec, que proporciona gran cantidad de sedimentos e imprime condiciones favorables para la formación de la playa cuando la barra del río se rompe durante la época de lluvias.

Se concluye que las zonas de mesoplaya y de supraplaya con arenas medias $(1 \phi)$ en La Escobilla resultaron ser las más adecuadas para 


\section{Desembocadura \\ del río}
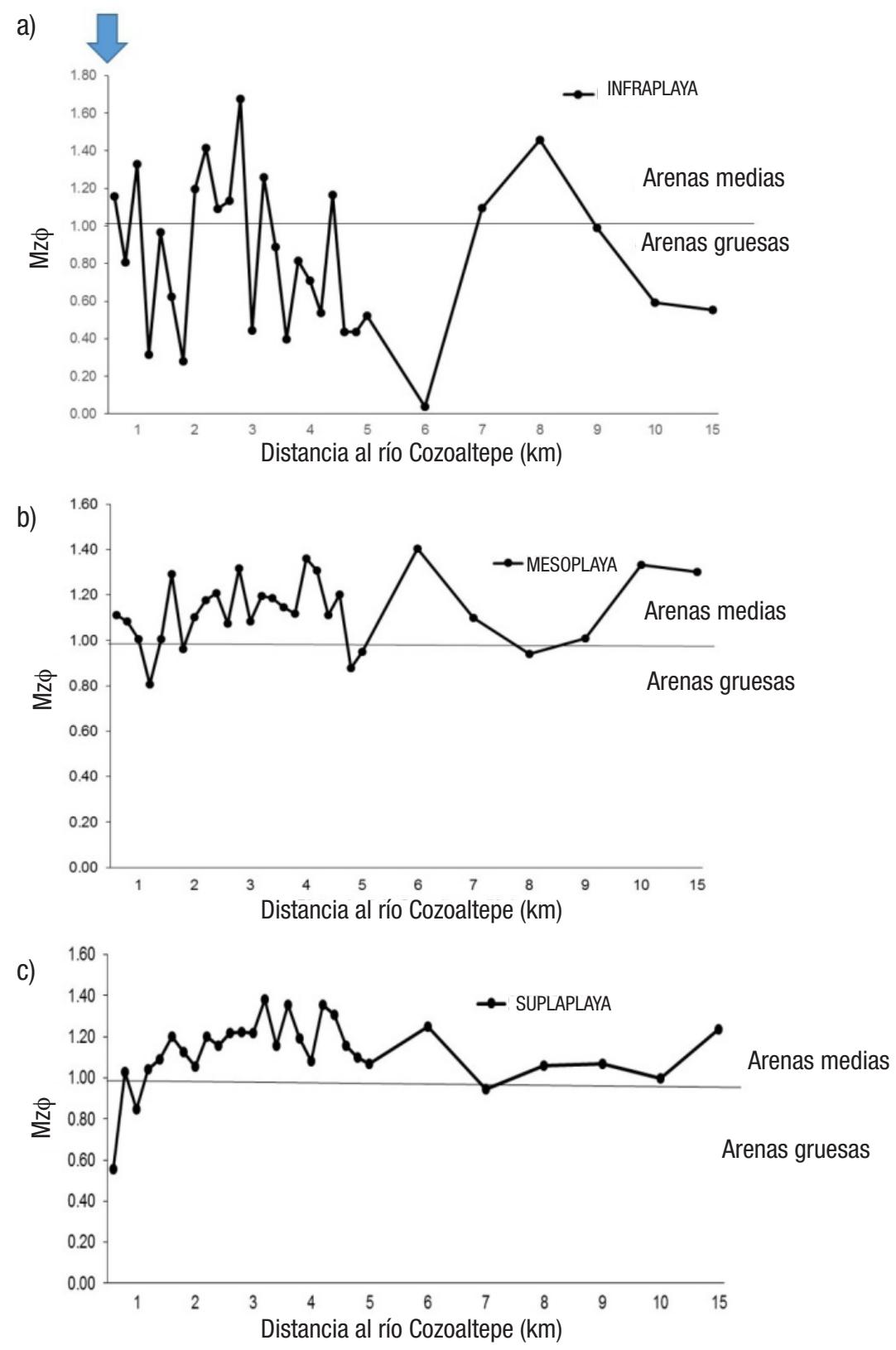

Figuras 7a-c. Distribución del tamaño gráfico promedio $(\mathrm{Mz} \phi)$ en las zonas de a) infraplaya, b) mesoplaya, y c) supraplaya a lo largo de la playa La Escobilla, Oaxaca, México.

las anidaciones de Lepydochelis olivacea, y se observó que las zonas con mayores anidaciones se encuentran a una distancia de $2.3 \mathrm{~km}$ hacia el este del río Cozoaltepec, donde se tiene la menor pendiente $\left(1^{\circ}\right)$ y el ancho de la playa es de $68 \mathrm{~m}$. Esto supone ser un lugar conveniente para el ascenso de las tortugas hacia su sitio de anidación en arenas de playa cuyo tamaño (arenas medias) permite que las tortugas excaven más fácilmente los sitios de anidación, pues si la arena es muy gruesa o muy fina resulta más difícil la excavación.
La disminución de la franja de sedimentos litorales por actividades antrópicas hace vulnerable al ambiente de playa como un sitio adecuado para las anidaciones, ya que se ve amenazada su ocurrencia. Por este motivo se recomienda no afectar los aportes naturales de sedimentos, pues tanto la textura de sedimentos como la morfología de la playa se afectarían en detrimento del éxito de la arribazón y anidación. 

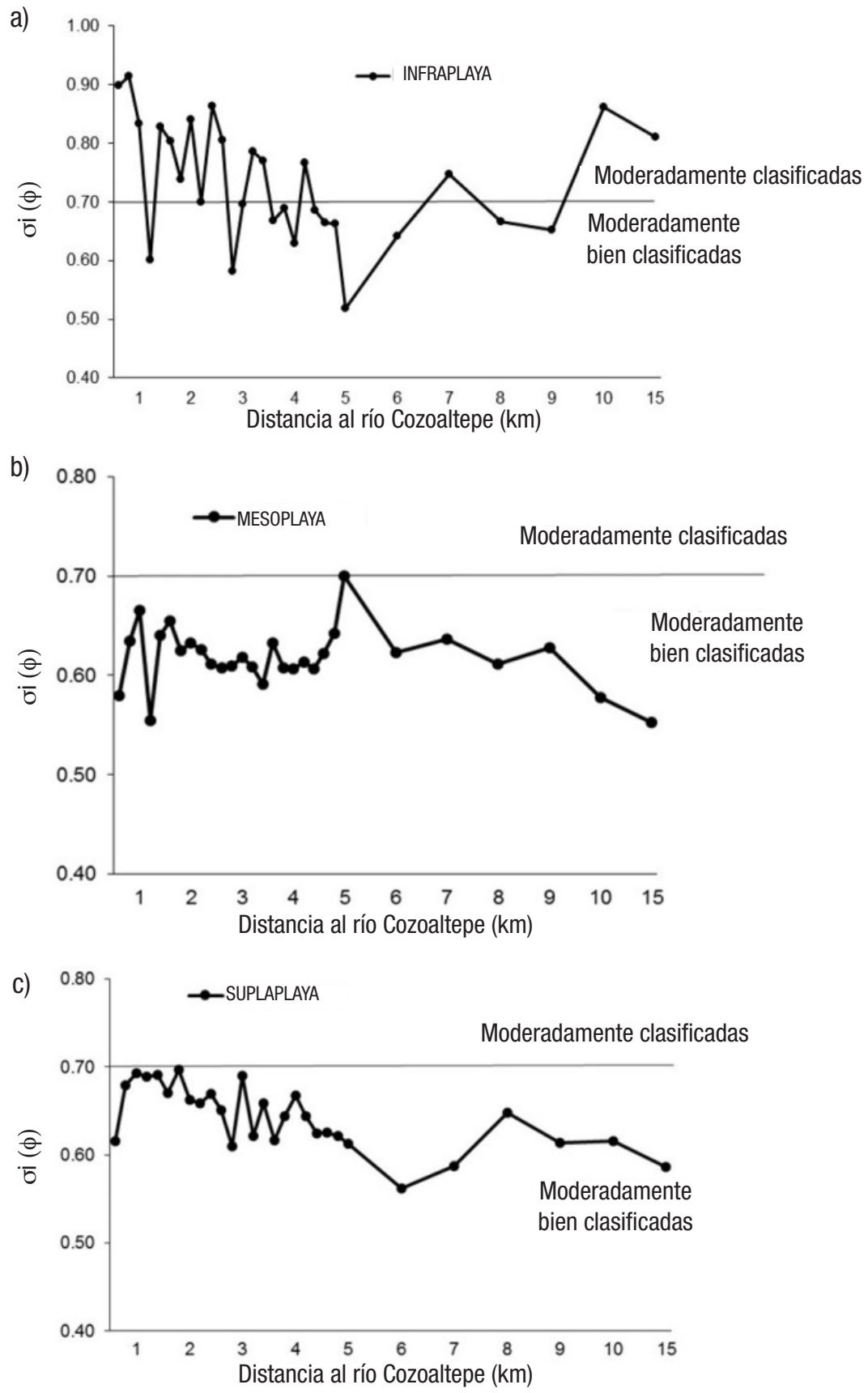

Figuras 8a-c. Distribución de la desviación estándar a partir de la desembocadura del río Cozoaltepec a lo largo de la playa La Escobilla, Oaxaca, México. a) Infraplaya; b) Mesoplaya; c) Supraplaya.

\section{AGRADECIMIENTOS}

Se agradece a las autoridades del Instituto de Ciencias del Mar y Limnología de la Universidad Nacional Autónoma de México (UNAM) y de la Comisión Nacional de Áreas Naturales Protegidas (CONANP) por las facilidades otorgadas para la realización de esta investigación durante su desarrollo. A la DGAPA por el apoyo al proyecto PAPIME PE106016.
Al CONACyT por su contribución en la formación de recursos humanos dentro del Posgrado de Ciencias del Mar y Limnología de la UNAM. Al Geog. Germán Gómez por su apoyo en los trabajos de campo, al M. en C. Eduardo Morales de la Garza y a Ricardo Martínez Domínguez por Ios análisis sedimentológicos, y al Lic. Sergio Macías Medrano por la realización de los mapas de localización y geología. 


\section{REFERENCIAS}

Ackerman, R. A. 1997. The nest environment and the embryonic development of sea turtles. In: Lutz, P.L. \& J. A. Musick (Eds.). The Biology of Sea Turtles. Boca Raton, Florida: CRC Press, pp. 83-106.

Albavera, P. E. 2006. Revisión de la anidación de tortugas marinas en los campamentos del Centro Mexicano de la Tortuga durante el periodo 2001-2005. Centro Mexicano de la Tortuga de la Comisión Nacional de Áreas Naturales Protegidas (CONANP).

Arriaga, C. L., D. E. Vázquez, C. J. González, R. R. Jiménez, L. E. Muñoz \& S. V. Agullar (Coords). 1998. Regiones marinas prioritarias de México. Comisión Nacional para el Conocimiento y Uso de la Biodiversidad, México. Disponible en línea en: http://www.conabio.gob.mx (consultado el 12 septiembre, 2014).

ÁvILA, J. B. 2006. Éxito de eclosión en la arribazón de la tortuga golfina Lepidochelys olivacea (Eschscholtz 1829) en la playa de La Escobilla, Oaxaca. Tesis de Licenciatura. Universidad del Mar, Puerto Ángel, Oaxaca.

Barik, S. K., P. K. Mohanty, P. K. Kar, B. Behera \& S. Patra. 2014. Environmental cues for mass nesting of sea turtles. Ocean and Coastal Management 95: 233-240. D0I:10.1016/j.ocecoaman.2014.04.018

Bolongaro, C. R. A., G. A. Z. Márquez, R. V. Torres \& V. A. García. 2010. Vulnerabilidad de sitios de anidación de tortugas marinas por efectos de erosión costera en el estado de Campeche. In: Botello, A.V., S. Villanueva-Fragoso, J. Gutiérrez \& J. L. Rojas-Galaviz (Eds.). Vulnerabilidad de las zonas costeras mexicanas ante el cambio climático. SEMARNAT-INE (Secretaria del Medio Ambiente Recursos Naturales y Pesca-Instituto Nacional de Ecología). UNAM-ICMyL. Universidad Autónoma de Campeche. pp. 73-96.

Carranza, E. A. 1984. Estudio Sedimentológico de Playas del Estado de Chiapas, México. Anales del Instituto de Ciencias del Mar y Limnología, UNAM, México 13 (1): 331-344.

Carranza, E. A. 2001. Grain size and sorting in modern beach sands. Journal of Coastal Research 17 (1): 38-52.

Carranza-Edwards, A. \& M. Caso Chávez. 1994. Zonificación del perfil de playa. Geo-UNAM 2 (2): 26-32.

Carranza, E. A., E. M. Gutiérrez \& T. R. RodríGuez. 1975. Unidades MorfoTectónicas Continentales de las Costas Mexicanas. Anales del Instituto de Ciencias del Mar y Limnología, UNAM, México 2 (1): 81-88.

Carranza, E. A., L. Rosales \& R. Lozano. 1987. Estudio sedimentológico de playas del estado de Oaxaca, México. Anales del instituto de Ciencias del Mar y Limnología, UNAM, México 15 (2): 23-38.

Carranza-Edwards, A., J. J. Kasper Zubillaga, L. Rosales-Hoz, E. A. Morales de la Garza \& R. Lozano Santa Cruz. 2009. Beach sand composition and provenance in a sector of the southwestern Mexican Pacific. Revista Mexicana de Ciencias Geológicas 26 (2): 433-447.

conanp (Comisión Nacional de áreas naturalmente Protegidas). 2008. Iniciativa con proyecto de decreto por el que se reforman diversos artículos de la ley general de vida silvestre. Disponible en línea en: http://www.conanp.gob.mx/centros_tortugas.html (consultado el 17 de octubre de 2015).
Davies, J. C. 1973. Statistics and Data Analysis in Geology. John Wiley and Sons, Nueva York. 550 p.

ECKert, S. A., K. L. ECKert, P. Ponganis \& G. L. KoOyman. 1989. Diving and foraging behaviour of leatherback sea turtles (Dermochelys coriacea). Canadian Journal of Zoology 67 (11): 2834-2840. D0I:10.1139/ z89-399

Eckert, K. L., K. A. Buorndal, F. A. Abreu-Grobols \& M. Donelly (Eds.). 1999. Research and Management Techniques for the Conservation of Sea Turtles. Prepared by IUCN/SSC (International Union for the Conservation of Nature/ Species Survival Comission) Marine Turtle Specialist Group. International Union for the Conservation of Nature, Gland, Switzerland. $248 \mathrm{p}$.

Foley, A. M., S. A. Peck \& G. R. Harman. 2006. Effects of sand characteristics and inundation on the hatching success of Loggerhead Sea Turtle (Caretta caretta) clutches on low-relief mangrove islands in southwest Florida. Chelonian Conservation and Biology 5 (1): 3241. D0I:10.2744/1071-8443(2006)5[32:EOSCAI]2.0.C0;2

Foley, A. M., B. A. Schroeder., R. Hardy., S. L. MacPherson \& M. Nicholas. 2014. Long-term behavior at foraging sites of adult female loggerhead sea turtles (Caretta caretta) from three Florida rookeries. Marine Biology 161 (6): 1251-1262. D0I:10.1007/s00227-014-2415-9

Folk, R. L. 1974. Petrology of sedimentary rocks. Hemphill Publishing Company, Austin Texas. 398 p.

Fuentes-Farias, A. L., V. H. Garduño-Monroy, G. Gutiérrez-Ospina, L. Pérez-Cruz, E. Meléndez-Herrera \& J. Urrutia-Fucugauchi. 2010. Reconnaissance study of Colola and Maruata, the nesting beaches of marine turtles along the Michoacan coast in southern Mexico. Geofísica Internacional 49 (4): 201-212. D0I:10.1016/j.ocecoaman.2014.04.018

Hays, G. C. 2004. Good news for sea turtles. Trends in Ecology and EvoIution 19 (7): 349-351.

Hawkes, L. A., A. C. Broderick, M. H. Godfrey \& B. J. Godley. 2009. Climate change and marine turtles. Endangered Species Research 7: 137154. DOI:10.3354/esr00198

IUCN (International Union for Conservation of Nature). 2015. The IUCN Red List of Threatened Species. Versión 2015-3. Disponible en línea en: http://www.iucnredlist.org (consultado el 14 de agosto de 2015).

Johannes, R. E. \& D. W. Rimmer. 1984. Some distinguishing characteristics of nesting beaches of the green turtle Chelonia mydas on North West Cape Peninsula, Western Australia. Marine Biology 83: 149-154.

KING, C. A. M. 1972. Beaches and Coasts. 2nd. edition. Arnold, London. $570 \mathrm{p}$.

KomaR, P. D. 1976. Beach processes and sedimentation. Prentice Hall, Inc. New Jersey. 429 p.

Lugo, H. J. 1990. El relieve de la República Mexicana. Revista del Instituto de Geología 9 (1): 82-111.

Mazaris, A. D., G. Matsinos \& J. D. Pantis. 2009. Evaluating the impacts of coastal squeeze on sea turtle nesting. Ocean and Coastal Management 52 (2): 139-145. DOI:10.1016/j.ocecoaman.2008.10.005 
MıLLER, J. D. 1997. Reproduction in sea turtles. In: Lutz, P.L. \& J.A. Musick (Eds.). The Biology of Sea Turtles. CRC, Boca Raton, Florida. pp. 51-81.

Mortimer, J. A. 1990. The Influence of Beach Sand Characteristics on the Nesting Behavior and Clutch Survival of Green Turtles (Chelonia mydas). Copeia 3: 802-817. D0I:10.2307/1446446

Mortimer, J. A. 1995. Factors influencing beach selection by nesting sea turtles. In: Bjordnal K. A. (Ed.). Biology and Conservation of Sea Turtles. Smithsonian Institution Press. pp. 45-51.

Mortimer, J. A. 2000. Sea turtle conservation programmes: Factors determining success or failure. In: Salm, R. V., J. R. Clark \& E. Siirila (Eds). Marine and Coastal Protected Areas: A guide for planners and managers IUCN. Washington, D.C. pp. 327-333.

Pethick, J. S. \& S. Crooks, S. 2000. Development of a coastal vulnerability index: a geomorphological perspective. Environmental Conservation 27 (4): 359-367. D0I:10.1017/S0376892900000412.

Poloczanska, E. S., C. J. Limpus \& G. C. Hays. 2009. Vulnerability of Marine Turtles to Climate Change. In: Sims, D.W. (Ed). Advances in Marine Biology 56: 151-211. D0I:10.1016/S0065-2881(09)56002-6

Pritchard, P. C. H. \& J. A. Mortimer. 2000. Taxonomía, morfología externa e identificación de las especies. In: Eckert, K. L., K. A. Bjorndal, F. A. AbreuGrobois \& M. Donnelly (Eds). 2000 (Traducción al español). Técnicas de Investigación y Manejo para la Conservación de las Tortugas Marinas. Grupo Especialista en Tortugas Marinas UICN/
CSE Publicación No. 4. Pennsylvania, USA. pp. 23-41. Disponible en línea en: https://portals.iucn.org/library/sites/library/files/ documents/1999-076-Es.pdf

ScHWARTZ, F. J. 1982. Correlations of nest sand asymmetry and percent loggerhead sea turtle nest hatch in North Carolina determined by geological sorting analyses. ASB Bulletin 29: 83.

Seminoff, J. A. \& K. Shanker. 2008. Marine turtles and IUCN Red Listing: a review of the process, pitfalls and novel assessment approaches. Journal of Experimental Marine Biology and Ecology 356 (1): 52-68. D0I:10.1016/j.jembe.2007.12.007

Servicio Geológico Mexicano. 2002. Carta geológico-minera: Puerto Escondido D14-3 (0axaca), escala 1:250,000. 1 hoja.

Yalcin-ÖZdilek, S., H. G. Özdilek \& F. Ozaner. 2007. Possible Influence of Beach Sand Characteristics on Green Turtle Nesting Activity on Samandag Beach, Turkey. Journal of Coastal Research 23 (6): 13791390. DOI:10.2112/06-0630.1

Wood, D. W. \& K. A. BJoRndAL. 2000. Relation of temperature, moisture, salinity, and slope to nest site selection in loggerhead sea turtles. Copeia 1: 119-128. D0I:10.1016/j.jembe.2007.12.007

Wyrtki, K. 1965. Surface currents of the eastern tropical Pacific Ocean. Inter-American Tropical Tuna Commission Bulletin 9: 271-304. 